\title{
NUMERICAL RANGES OF THE PRODUCT OF OPERATORS
}

\author{
Hongke Du, Chi-Kwong Li, Kuo-Zhong Wang, \\ YUEQING WANG AND NING ZUO
}

\begin{abstract}
We study containment regions of the numerical range of the product of operators $A$ and $B$ such that $W(A)$ and $W(B)$ are line segments. It is shown that the containment region is equal to the convex hull of elliptical disks determined by the spectrum of $A B$, and conditions on $A$ and $B$ for the set equality holding are obtained. The results cover the case when $A$ and $B$ are self-adjoint operators extending the previous results on the numerical range of the product of two orthogonal projections.
\end{abstract}

Mathematics subject classification (2010): 15A60, 47A12.

Keywords and phrases: Numerical range, product of matrices and operators.

\section{REFERENCES}

[1] H. KLAJA, The numerical range and the spectrum of a product of two orthogonal projections, Journal of Mathematical Analysis and Applications 411 (2014), 177-195.

[2] Y. Q. WANG, H. K. DU, A characterization of maximum norms of commutators of positive contractions, Journal of Mathematical Analysis and Applications 348 (2) (2008), 990-995.

[3] T. Ogasawara, A Theorem on operator algebras, J. Sci. Hiroshima Univ. Ser. A., 18 (1955), $307-$ 309.

[4] K. Gustafson And D. K. M. RaO, Numerical range. The field of values of linear operators and matrices, New York: Springer, 1997.

[5] R. A. Horn AND C. R. Johnson, Topics in matrix analysis, Cambridge: Cambridge University Press, 1991

[6] P. R. Halmos, A Hilbert space problem book, 2nd ed., New York: Springer, 1982.

[7] I. SPITKOVSKY, On polynomials of two projections, Electronic Journal of Linear Algebra, vol. 15, pp. 154-158. 